\title{
Isotropic Sequence Order Learning
}

\author{
Bernd Porr \\ bp1@cn.stir.ac.uk \\ Florentin Wörgötter \\ worgott@cn.stir.ac.uk \\ Department of Psychology, University of Stirling, Stirling FK9 4LA, Scotland
}

In this article, we present an isotropic unsupervised algorithm for temporal sequence learning. No special reward signal is used such that all inputs are completely isotropic. All input signals are bandpass filtered before converging onto a linear output neuron. All synaptic weights change according to the correlation of bandpass-filtered inputs with the derivative of the output. We investigate the algorithm in an open- and a closed-loop condition, the latter being defined by embedding the learning system into a behavioral feedback loop. In the open-loop condition, we find that the linear structure of the algorithm allows analytically calculating the shape of the weight change, which is strictly heterosynaptic and follows the shape of the weight change curves found in spike-time-dependent plasticity. Furthermore, we show that synaptic weights stabilize automatically when no more temporal differences exist between the inputs without additional normalizing measures. In the second part of this study, the algorithm is is placed in an environment that leads to closed sensormotor loop. To this end, a robot is programmed with a prewired retraction reflex reaction in response to collisions. Through isotropic sequence order (ISO) learning, the robot achieves collision avoidance by learning the correlation between his early range-finder signals and the later occurring collision signal. Synaptic weights stabilize at the end of learning as theoretically predicted. Finally, we discuss the relation of ISO learning with other drive reinforcement models and with the commonly used temporal difference learning algorithm. This study is followed up by a mathematical analysis of the closed-loop situation in the companion article in this issue, "ISO Learning Approximates a Solution to the Inverse-Controller Problem in an Unsupervised Behavioral Paradigm" (pp. 865-884).

\section{Introduction}

A central goal of every autonomous agent is to maintain homoeostasis (Ashby, 1956), without which it will eventually disintegrate ("die"). A generic way to achieve this is by reacting to a disturbance of the homoeostasis with a closed-loop negative feedback mechanism (a reflex), which will 
compensate for the disturbance by means of a (motor) reaction. Thus, the simplest form of sensible autonomous behavior can be obtained by designing an agent whose (re-)actions are reflex based (Brooks, 1989). This type of behavior is found even in rather primitive animals like amoebas, which retract their philopodia when encountering a potentially damaging chemical gradient.

Such sensor-motor reflex loops represent typical feedback reaction systems, because a reflex will always be elicited only after a sensor event has already been encountered, as the word feedback implies. The reaction delay, which is unavoidably associated with every reflex loop, can even lead to fatal situations in the worst case. Thus, in any kind of improved behavior, the acting agent will try to avoid reflexes, for example, by predicting one sensor event from another earlier occurring event (at a different sensor). This takes place when predicting pain from the heat that radiates from a hot surface in order to prevent a retraction reflex by means of an anticipatory avoidance reaction. In this example, heat radiation and pain are causally related. Many other similar causal relations exist during the life of an animal, for example, between smell and taste when foraging or between vision and touch when exploring. In all of these cases, a temporal sequence of sensor events occurs, which needs to be learned in order to avoid reflex reactions to the later event. Thus, temporal sequence learning is a dominant aspect of animal behavior. It requires a late event, which serves as a reference to which the earlier event temporally relates. The goal is to learn this specific temporal relation and turn reactive into proactive behavior.

In artificial systems, temporal sequence learning can be achieved, for example, by classical Hebbian learning (Hebb, 1949) in combination with delays (Levy \& Minai, 1993), by differential Hebbian learning (Kosco, 1986; Klopf, 1986), or by the very influential temporal difference (TD) reinforcement learning algorithm (Sutton, 1988; Montague, Dayan, \& Sejnowski, 1993; Dayan \& Sejnowski, 1994; Abbott \& Blum, 1996; Dayan, Kakade, \& Montague, 2000; Rao \& Sejnowski, 2001; Haruno, Wolpert, \& Kawato, 2001; Schultz \& Suri, 2001). In TD learning, the "later event" is represented by a designated reference signal (mostly a reward or punishment signal) to which the prediction of the learner is explicitly compared. The reference signal thus represents an explicitly defined so-called evaluative feedback for the learning, which stops when prediction and reward match. This may pose a problem, as pointed out by Klopf (1988), who had emphasized that evaluative feedback cannot exist in autonomously acting agents, which normally cannot rely on any external, evaluative, (teacher-like) signal. Klopf's differential Hebbian algorithm is indeed nonevaluative and belongs to the so-called class of drive reinforcement models. This issue, however, is still rather controversial. Klopf's arguments are convincing, yet evidence exists that dopamine could indeed serve as such a possible reward-like reference signal in the brains of higher mammals (Schultz, Dayan, \& Montague, 1997; Schultz \& Suri, 2001), which can respond to complex learning situations 
such as instrumental (operand) conditioning. Less complex forms of learning such as basic classical conditioning, however, can be observed even in very simple creatures (for example, Aplysia), which do not have a reward system (Kandel et al., 1983).

One aspect of the current study, therefore, is to design an algorithm in which sequence order learning takes place in a reward-free, unsupervised way by means of a temporal Hebb learning rule that is isotropic with respect to the inputs (hence the name ISO learning, which stands for isotropic sequence order learning). ${ }^{1}$ Thus, the algorithm is strictly based on the causal relation between its inputs, which is in reality often given by the "properties of the world," as described by the examples above. The reference signal is just the latest occurring signal (which often has the highest initial synaptic weight), a situation that can change during learning.

The article is organized in the following way. First, we introduce the algorithm in an open-loop paradigm. Its linear structure allows an analytical treatment of some of its main characterizing features. More complex aspects are addressed with simulations. In this part of the study, it will become clear that all input lines are mathematically equivalent in our algorithm. Furthermore, we will show that the algorithm performs strict heterosynaptic learning. A detailed comparison of ISO learning with other algorithms is given in appendix $\mathrm{B}$.

In the second part of this study we embed our algorithm in a behavioral loop by means of a robot experiment. This creates a self-referential system and leads to stability. As a consequence of the fact that learning is heterosynaptic, we find that synaptic weights will self-stabilize as soon as the reflex input becomes silent.

One central aspect of this and the companion article, "ISO Learning Approximates a Solution to the Inverse-Controller Problem in an Unsupervised Behavioral Paradigm," is to show that unsupervised open-loop ISO learning inherently turns into a reference-based system as soon as it is embedded into a nonevaluative environment that leads to a closed sensor-motor loop. This could be expected from the results of Klopf (1988), but we will show analytically in the companion article that such a closed-loop system creates, by means of the learning process, a forward model of its environment. Temporal sequence learning using the ISO learning algorithm can therefore be understood as finding a solution to the specific inverse controller problem that replaces a reflex by its forward model.

${ }^{1}$ The self-referential structure of this abbreviation is meant to hint at the self-referential behavioral loop introduced in the second part of this article. 


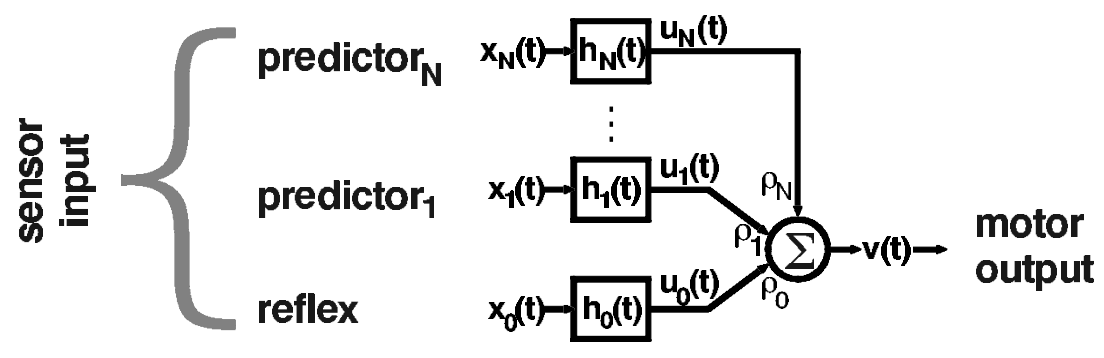

Figure 1: The basic circuit in the time domain.

\section{Open Loop: ISO Learning}

First, we describe the algorithm itself and its characteristics without behavioral feedback.

We consider a system of $N+1$ linear filters $h$ receiving inputs $x$ and producing outputs $u$. The filters connect with corresponding weights $\rho$ to one output unit $v$ (see Figure 1).

In section 1, we emphasized that all input lines of our algorithm are mathematically equivalent. It should be remembered, however, that functionally, many times there are distinctive differences among them. As a consequence, we will use $x_{0}$ to denote the one unit that will later represent the reflex pathway. This has no mathematical consequences and is done only for convenience. The output $v$ is then given as

$$
v=\rho_{0} u_{0}+\sum_{k=1}^{N} \rho_{k} u_{k} .
$$

Learning (weight change) takes place according to a differential Hebb rule,

$$
\frac{d}{d t} \rho_{j}=\mu u_{j} v^{\prime} \quad \mu \ll 1,
$$

where the weight change depends on the correlation between $u_{j}$ and the derivative of $v$. An extensive discussion how this rule relates to TD learning and to other differential Hebbian learning rules, as introduced by Klopf $(1986,1988)$ and Kosco $(1986)$, is given in section 4 and appendix B. Here, we note only that other differential Hebbian learning rules use filtering and derivates in different pathways as compared to ISO learning (see Figure 12 for circuit diagrams).

All weights can change (also $\rho_{0}$ ). The constant $\mu$ is adjusted such that all weight changes occur on a much longer timescale (i.e., very slowly) as 
compared to the decay of the responses $u$. Thereby the system operates in the steady-state condition.

In general, the system that we consider shall operate in continuous time (e.g., with neuronal rate codes), and it shall be able to handle continuous input functions $x(t)$ of arbitrary shape.

The transfer function $h$ shall be that of a bandpass that transforms a $\delta$ pulse input into a damped oscillation (see Figure 2A) and is specified in the Laplace domain,

$$
h(t) \leftrightarrow H(s)=\frac{1}{(s+p)\left(s+p^{*}\right)},
$$

where $p^{*}$ represents the complex conjugate of the pole $p=a+i b$. It is important to note that such a bandpass is stable only if its pole pair is located on the left complex half-plane; otherwise, an amplified oscillation is obtained.

Real and imaginary parts of the poles are given by

$$
\begin{aligned}
& a:=\operatorname{Re}(p)=-\pi f / Q \\
& b:=\operatorname{Im}(p)=\sqrt{(2 \pi f)^{2}-a^{2},}
\end{aligned}
$$

where $f$ is the frequency of the oscillation. The damping characteristic of the resonator is reflected by $Q>0.5$. Small values of $Q$ lead to a strong damping.

The use of resonators (bandpass filters) is motivated by biology because oscillatory neuronal responses (Traub, 1999) and bandpass-filtered response characteristics (at virtually all sensory front ends, cell membranes [Shepherd, 1990], and ion channels like NMDA) are very prevalent in neuronal systems. Several examples for the utilization of such bandpass-filtered responses provide Grossberg and Schmajuk (1989) with their spectral timing model, which they have used in different applications (Grossberg, 1995; Grossberg \& Merrill, 1996).

Thus, the main idea is to use a neuron that gets bandpass-filtered sensor signals at its inputs and generates a motor output. Later, one of these bandpasses $\left(h_{0}\right)$ has the special task of providing the input for a reflex-like reaction. The other bandpass-filtered sensor signals are candidates for generating an earlier motor reaction through learning.

\subsection{Analytical Findings: Open-Loop Condition.}

2.1.1 Timing Dependence of Weight Change. Here we address the question how the timing between the input signals influences the weight change. In order to perform analytical calculations, we introduce two restrictions, 
a

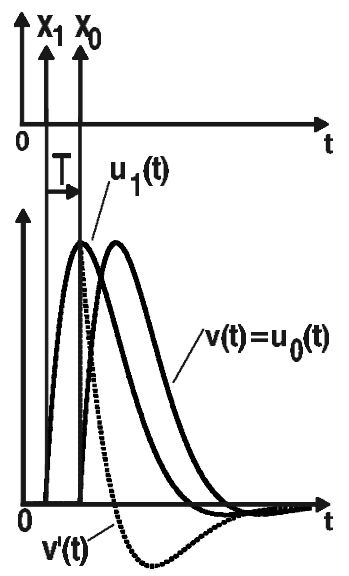

b

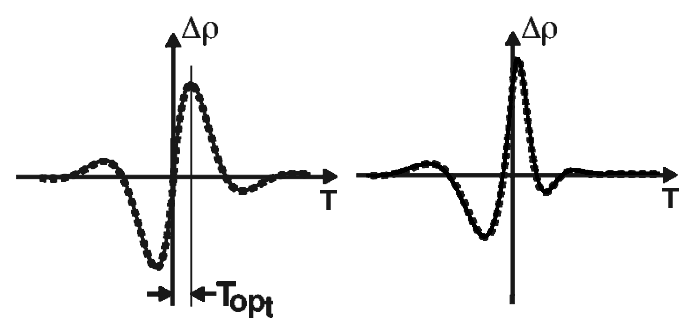

d

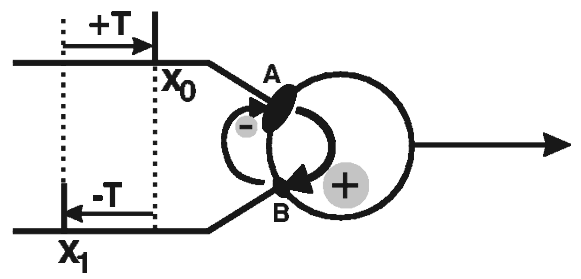

Figure 2: Input functions and the initial weight change for $t=0$ according to equations 2.13 and 2.14. (a) The inputs $x$, the impulse responses $u$ for a choice of two different resonators $h$, and the derivative of the output $v^{\prime}$. (b) The initial weight change $\rho_{1}(T)_{t=0}$ for $H_{1}=H_{0}, Q=1, f=0.01$ (arbitrary units) and (c) for resonators with different frequencies $f_{0}=0.01, f_{1}=0.02$ but with the same $Q=1$. The solid lines in $b$ and $c$ represent the analytical solutions derived from equations 2.13 and 2.14, and the dots the simulation results from the numerical integration of equation 2.9 with the same parameters for $f$ and $Q$. For that purpose, the two filters $H_{0}$ and $H_{1}$ get two different inputs $x_{1}(t)=\delta(t)$ and $x_{0}(t)=\delta(t-T)$. This pulse sequence was repeated every 2000 time steps. After 400,000 time steps, the weight $\rho$ was measured and plotted against the temporal difference $T$. The learning rate was set to $\mu=0.001$. (d) Schematic explanation of the mutual weight change at a strong (A) and a weak synapse (B) with two subsequent delta pulses at the inputs $x_{1}$ and $x_{0}$. For further explanations, see the text.

which we use often throughout the theoretical parts of this article:

1. We will consider only two resonators, thus, $N=1$.

2. Accordingly we have to deal with only two input functions $x_{0}, x_{1}$, and we define them as (delayed) $\delta$-pulses:

$$
\begin{aligned}
& x_{0}(t)=\delta(t-T), \quad T \geq 0 \\
& x_{1}(t)=\delta(t) .
\end{aligned}
$$

The first restriction is necessary because the analytical treatment of the case $N>1$ is very intricate and largely impossible. Concerning the second 
restriction, we note that the theory of signal decomposition allows composing any causal input function from $\delta$-pulses. Thus, the second constraint is not really a restriction.

The delay $T$ ensures a well-defined causal relation between both inputs, where $x_{0}$ (the latter of the two) is the timing reference (the reflex input). Especially the section on the robot implementation will show that the algorithm (with $N>1$ ) is very robust with respect to variations in $T$.

In general, we use as an initial condition $\rho_{0}=1$ and $\rho_{1}=0$. For the analytical treatment, we consider only the weight change at $\rho_{1}$. (In fact, we later show that the algorithm normally operates always in a domain where $\rho_{0}$ changes very little.)

Because we assume steady state, we can rewrite the product in the learning rule (see equation 2.2) as a correlation integral between input and output:

$$
\begin{aligned}
\rho_{1} & \rightarrow \rho_{1}+\Delta \rho_{1} \\
\Delta \rho_{1}(T) & =\mu \int_{0}^{\infty} u_{1}(T+\tau) v^{\prime}(\tau) d \tau .
\end{aligned}
$$

Similar to other approaches (Oja, 1982), we compute the weight change for the initial development of the weights as soon as learning starts, because this is indicative of the continuation of the learning. Therefore, we assume $\rho_{1}(t)=0$ for $t=0$, and equation 2.9 turns into

$$
\rho_{1}(T)_{t=0}=\mu \int_{0}^{\infty} u_{1}(T+\tau) u_{0}^{\prime}(\tau) d \tau .
$$

In simple cases (e.g., for $h_{0}=h_{1}$ ), this integral can be solved directly. A general solution, which can be extended to cover more than two inputs, requires applying the Laplace transform using the notational convention $x(t) \leftrightarrow X(s)$ for a transformation pair of functions in the time and the Laplace domain.

The linearity of our system allows solving the integral in equation 2.10 analytically, which is possible with the help of Plancherel's theorem (see appendix $\mathrm{A}$ for this rather unknown theorem). Applying it together with the shift theorem $x\left(t-t_{0}\right) \rightarrow X(s) e^{-t_{0} s}$ to equation 2.10, we get:

$$
\begin{aligned}
\Delta \rho_{1} & =\mu \frac{1}{2 \pi} \int_{-\infty}^{+\infty} H_{1}(-i \omega)\left[i \omega e^{-T i \omega} H_{0}(i \omega)\right] d \omega \\
& =\mu \frac{1}{2 \pi} \int_{-\infty}^{+\infty} H_{1}(i \omega)\left[-i \omega e^{T i \omega} H_{0}(-i \omega)\right] d \omega .
\end{aligned}
$$

Note that the symmetry of Plancherel's theorem is broken due to the exponential term. Equation 2.11 represents a Fourier transform, and equation 2.12 is its inverse. Both integrals can be evaluated with the method of 
residuals. Equation 2.12, however, offers the advantage that we can neglect the right complex half-plane, because it leads to contributions for negative time (i.e., $t<0$ ) only (McGillem \& Cooper, 1984; Stewart, 1960). Thus, of the four residuals (poles) for $H_{1}$ and $H_{0}$, only those of $H_{1}$ need to be considered because those of $H_{0}$ have flipped their sign in equation 2.12 and appear now on the right complex half-plane. We get as the final result,

$$
\begin{array}{ll}
\rho_{1}(T)_{t=0}=\mu \frac{b_{1} M \cos \left(b_{1} T\right)+\left(a_{1} P+2 a_{0}\left|p_{1}\right|^{2}\right) \sin \left(b_{1} T\right)}{b_{1}\left(P+2 a_{1} a_{0}+2 b_{1} b_{0}\right)\left(P+2 a_{1} a_{0}-2 b_{1} b_{0}\right)} e^{-T a_{1}} & T \geq 0 \\
\rho_{1}(T)_{t=0}=\mu \frac{b_{0} M \cos \left(b_{0} T\right)+\left(a_{0} P+2 a_{1}\left|p_{0}\right|^{2}\right) \sin \left(b_{0} T\right)}{b_{0}\left(P+2 a_{0} a_{1}+2 b_{0} b_{1}\right)\left(P+2 a_{0} a_{1}-2 b_{0} b_{1}\right)} e^{-T a_{1}} & T<0,
\end{array}
$$

where $M=\left|p_{1}\right|^{2}-\left|p_{0}\right|^{2}$ and $P=\left|p_{1}\right|^{2}+\left|p_{0}\right|^{2}$. If we assume identical resonators $H_{0}=H_{1}=H$, we get

$$
\Delta \rho_{1}(T)_{t=0}=\mu \frac{1}{4 a b} \sin (b T) e^{-a T},
$$

which is identical to the impulse response of the resonator itself apart from a different scaling factor.

The corresponding weight change curves are plotted in Figures $2 b$ and 2c. The curves show that synaptic weights are strengthened if the presynaptic signal arrives before the postsynaptic signal, and vice versa. The biological relevance of the learning curves becomes especially clear in the case $H_{0}=H_{1}$. This learning curve with identical resonators is similar to the curves obtained in neurophysiological experiments exploring spike-timingdependent synaptic plasticity (STDP or temporal Hebb; Markram, Lübke, Frotscher, \& Sakman, 1997; Bi \& Poo, 1998; Zhang, Tao, Holt, Harris, \& Poo, 1998; Abbott \& Nelson, 2000; Fu et al., 2002). ${ }^{2}$ Furthermore we find for this case (see Figure $2 b$ ) that the location of the maximum of the learning curve $T_{\text {opt }}$ falls in the interval

$$
\frac{\lambda}{2 \pi}<T_{\text {opt }}<\frac{\lambda}{4}, \quad \frac{1}{2}<Q<\infty,
$$

where $\lambda=1 / f$ is the wavelength of the resonator.

The isotropic setup of the algorithm in principle also leads to weight changes at $\rho_{0}$. It is, however, evident that the change in $\rho_{0}$ is (very) small when the contribution from the other inputs $\rho_{k}, k \geq 1$ is small. This is most easily seen when considering Figure $2 \mathrm{~d}$, which shows a situation that arises

${ }^{2}$ In order to reproduce STDP in a biophysical model, the signals $x$ and $u$ require a different interpretation involving NMDA conductances and backpropagating action potentials. This is the topic of a follow-up study currently in preparation (Saudargiene, Porr, \& Wörgötter, 2003). 
after some learning by using the standard initial conditions. The size of the synapses depicts the momentarily existing weight values. The input sequence is such that a weight increase arises at synapse $B$ from the influence of input line A onto line B ( $+T$ in learning curve), whereas weight decrease occurs at synapse A due to the inverse causal $(-T)$ influence of input line $B$ onto line A. The degree of change is depicted by the plus and minus signs, showing that the decrease of $\mathrm{A}$ is smaller than the increase of B. For two similar inputs, a simple rule of thumb is that the weight change $\Delta \rho$ roughly follows the weight value of the other input scaled by the learning rate $\mu$, while the sign of the change depends on the temporal sequence of events:

$$
\begin{aligned}
\Delta \rho_{\text {late input }} & \approx \mu \rho_{\text {early input }} \\
\Delta \rho_{\text {early input }} & \approx-\mu \rho_{\text {late input }} .
\end{aligned}
$$

As a result, the strong input roughly maintains its strength while the contributions from the other inputs are small. This is the typical case when learning is guided by a strong reflex and the organism has the task of building up predictive pathways that should be weaker but more precise in order to prevent the disturbance.

We note that the above analytical results can be extended to cover the most general system structure as represented in Figure 1 with $N>1$. Equation 2.1 turns into

$$
V(s)=\sum_{k=0}^{N} \rho_{k} U_{k}(s),
$$

keeping it in the Laplace domain, because then we can directly obtain

$$
\Delta \rho_{j}(T)=\mu \frac{1}{2 \pi} \int_{-\infty}^{+\infty}-i \omega V(-i \omega) U_{j}(i \omega) d \omega,
$$

which is the general form of equation 2.9 in the Laplace domain. It should be noted that for all $\Delta \rho_{j}$, this integral can still be evaluated analytically in the same way as in the special case with two resonators. In the following equations, we will always use the index $j$ for the input weights and $k$ for the summation of the output signal $v$.

2.1.2 Weight Change When $x_{0}$ Becomes Zero. In this section, we address the question of weight development when the reference input (reflex) becomes silent $\left(x_{0}=0\right)$ at some point during learning. This is motivated by the cases discussed in section 1, where the goal of learning is to avoid (late, painful, damaging) reflex reactions. Thus, setting $x_{0}=0$ corresponds to the condition when the reflex has successfully been avoided. Note that we are now left with just one input $\left(x_{1}\right)$ asking if its synaptic weight will continue 
to change. This would correspond to a situation of homosynaptic learning (e.g., homosynaptic long-term potentiation; Guo-Quing \& Poo, 1998). This section will show that our algorithm does not perform homosynaptic learning. Instead, the synaptic weight of $x_{1}$ stabilizes as soon as $x_{0}=0$. Thus, ISO learning is purely heterosynaptic learning.

We use the same two restrictions as above and start with equation 2.20, inserting equation 2.19 into it. We set $x_{0}=0 \leftrightarrow X_{0}=0$, and the weight change becomes

$$
\Delta \rho_{j}=\mu \frac{1}{2 \pi} \sum_{k=1}^{N} \rho_{k} \int_{-\infty}^{+\infty}-i \omega H_{k}(-i \omega) H_{j}(i \omega) d \omega .
$$

For $N=1$, we get

$$
\begin{aligned}
\Delta \rho_{1} & =\mu \frac{1}{2 \pi} \rho_{1} \int_{-\infty}^{+\infty}-i \omega H_{1}(-i \omega) H_{1}(i \omega) d \omega \\
& =-\mu \frac{i}{2 \pi} \rho_{1} \int_{-\infty}^{+\infty} \omega\left|H_{1}(i \omega)\right|^{2} d \omega .
\end{aligned}
$$

$H_{1}(i \omega) H_{1}(-i \omega)=|H(i \omega)|^{2}$ is valid since the transfer functions can always be expressed as products of complex conjugate pole pairs. Multiplying $H_{1}(i \omega)$ with $H_{1}(-i \omega)$ leads to products of a complex number with its conjugate counterpart, which renders its absolute value.

Since all transfer functions are symmetrical in relation to the real axis, the frequency response $|H(i \omega)|^{2}$ is also symmetrical, which leads to symmetrical responses in equation 2.23 at $\left|H_{1}(i \omega)\right|^{2}$. Due to $\omega$ in equation 2.23, the entire integral becomes antisymmetrical and thus zero. ${ }^{3}$ Thus, the weights stabilize if only $x_{1}$ is active.

This result can be summarized in a rather intuitive way: With $N=1$ and $x_{0}=0$, there is an input signal only at $x_{1}$. The weight change in that case is a correlation of a damped sine wave with its derivative, which is a damped cosine wave. The correlation of a sine with a cosine is always zero.

We have not attempted to calculate the behavior of the weights for $N>1$, which is very tedious, if not impossible. Instead, we will show simulation results for this later. However, the above argument can be extended by the Fourier theorem of wave decomposition to more inputs because each sine wave from a resonator is multiplied by its cosine counterpart. Thus, we also expect for $N>1$ a zero correlation and a stop of the weight development as soon as $x_{0}=0$.

${ }^{3}$ In a practical application (e.g., a digital infinite impulse response filter), this is true only if the frequency responses of the input $X_{1}$ and the transfer function $H_{1}$ vanish for high frequencies to avoid the integral's becoming ill defined $(\infty-\infty)$. In other words, the transfer functions must contain a low-pass term. This reflects the aspect that the time course of the input functions must be predictable (Kalman filter property). 

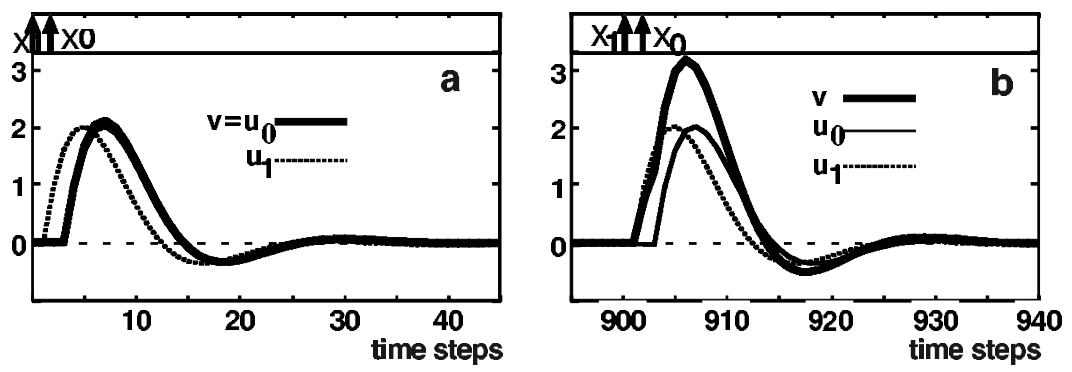

Figure 3: Simulation results with a circuit with two inputs, hence $N=1$ (see Figure 1). Input pulse sequences were repeated every 100 time steps, the first starting at zero. Both resonators had values of $Q_{0,1}=1$ and $f_{0,1}=0.1$. The other parameters were $\mu=0.01$ and $T=2$. Results for (a) $t=0$ and (b) $t=900$.

2.2 Simulations: Open-Loop Condition. In this section, we perform simulations with the neuronal circuit from Figure 1. The simulations have the purpose of validating the theoretical results from the previous section and exploring more complex situations (especially $N>1$ ) that are not analytically treatable.

The simulations were performed under Linux on an Athlon processor using $\mathrm{C}++$. The resonators were implemented as time-discrete infinite impulse response filters in the $z$-domain. We used the impulse invariant transformation from the $s$-plane to the $z$-plane and calculated the coefficients for the filters according to McGillem and Cooper (1984). We used normalized time steps resulting in normalized filter frequencies in the range $f=[0, \ldots, 0.5]$. In all applications, we used frequencies less than or equal to $f_{\max }=0.1$ in order to avoid sampling artifacts.

2.2.1 One Filter in the Predictive Pathway: $N=1$. As before, we begin with the simplest case, $N=1$ : one resonator in the reflex pathway $x_{0}$ and one resonator in the predictive pathway $x_{1}$, and use both restrictions $(1,2)$ noted previously.

Signal shape. Figure 3 a shows for $t=0$ the $\delta$-pulses at $x_{0,1}$ and the responses $u_{0}$ and $u_{1}$ from the resonators $H_{0}$ and $H_{1}$, respectively. Before learning, the output $v$ is identical to the signal $u_{0}$ because the weights were set to $\rho_{0}=1$ and $\rho_{1}=0$. The actual weight change of $\rho_{1}$ is caused by repeated pairing of the $\delta$-pulses at $x_{0}$ and $x_{1}$. The result after nine pairings is depicted in Figure $3 \mathrm{~b}$. The comparison between Figures $3 \mathrm{a}$ and $3 \mathrm{~b}$ shows that the onset of the output $v$ has shifted toward the earlier event $x_{1}$. Before learning, it was identical to the resonator response $u_{0}$ in the reflex pathway. After learning, the output is a superposition of both signals $u_{0,1}$, which leads to an onset that occurs together with the early onset of $u_{1}$. 
Thus, the circuit is able to "detect" the $\delta$-pulse at $x_{1}$ as a predictor of the $\delta$-pulse $x_{0}$.

Learning curve. Using the same setup, we can vary the interval $T$ and plot the change of $\rho_{1}$ in dependence of $T$ for the initial learning step (i.e., for $t=0$ after one correlation). This was simulated using identical resonators $H_{0}=H_{1}$ but also with different resonators $H_{0} \neq H_{1}$. The results are shown together with the analytical findings in Figures $2 b$ and $2 c$, having used the same parameters in both the simulation and the analytical calculation. Thus, the analytically calculated weight change curves are reproduced by the simulation results.

Development of $\rho_{0}$. In all cases discussed so far, both weights were allowed to change, and substantial changes in $\rho_{1}$ were found for about 10 to 50 pairings, while we have claimed that $\rho_{0}$ remains stable. An easy intuition why this basically holds can be gained by using the rule of thumb defined in equations 2.17 and 2.18. From this, it is clear that the change of $\rho_{0}$ remains tiny for a prolonged time in our setup because $\rho_{1}$ equals zero at the beginning and $\mu$ is very small. In simulations, we found that $\rho_{0}$ starts to change by more than $1 \%$ only after about 50,000 learning steps when using a standard learning rate of $\mu=0.001$ and $\rho_{1}=0, \rho_{0}=1$ as the usual initial conditions. Note that in the robot experiments shown later, the learning goal is reached after not more than 20 pairings. During this time, the change in $\rho_{0}$ is minuscule.

Several other relevant cases could occur.

- Another interesting initial condition would be setting the weights to the same initial values (e.g., $\rho_{0}=\rho_{1}=0.5$ ). This will still lead to a weight growth at $\rho_{1}$ (until about learning step 100,000), but now $\rho_{0}$ will drop from the beginning. Functionally, this could be interpreted as a situation where the reflex input becomes weaker, while the anticipatory pathway continues to take over. This could reflect a situation where the reflex has not been used for a long time, because then it is reasonable to allow the reflex to disappear, leading to $\rho_{0}=0$. The only measure that has to be taken is to stop the weight from changing its sign by keeping it at zero.

- In conditions where the reflex saves the organism from life-threatening situations, the weight $\rho_{0}$ can always be set to a fixed value.

- In conditions where we have multiple synaptic weights of similar strength (i.e., $N>1$ ), we can expect that the system's development will be dominated by stimulus-sequence-induced symmetry-breaking effects. This can lead to rather complex patterns, which would require a more detailed analysis (and is beyond the scope of this article). 

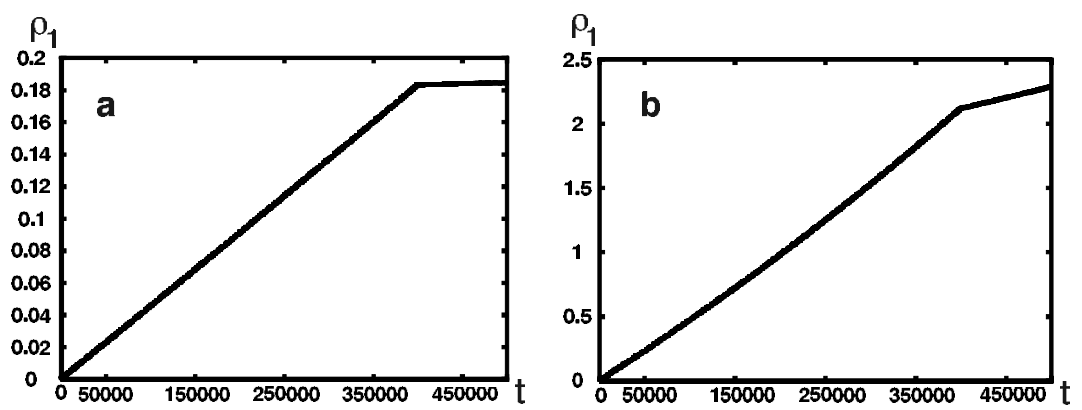

Figure 4: Simulated development of the weight $\rho_{1}$ for the case of two inputs $(N=1)$. Parameters were $f_{0,1}=0.01$ and $Q_{0,1}=1$. The inputs are triggered at a temporal difference of $T=15: x_{0}=\delta(t-T)$ and $x_{1}=\delta(t)$. The pairing of the delta pulses is repeated every 2000 time steps. The learning rate is set to (a) $\mu=0.001$ and (b) $\mu=0.01$.

Weight stabilization for $x_{0}=0$. The analytical results (see equation 2.22) predict that $\rho_{1}$ should stabilize as soon as $x_{0}=0$. This, however, also requires that the learning rate $\mu$ is zero, which in reality cannot be ultimately achieved. The following simulation results show the effect of the learning rate on the development of the weights and compare the analytically obtained result with those obtained for more realistic situations. The simulation to test this was performed in the following way. First, we triggered the two resonators with paired $\delta$-pulses. Then the input $x_{0}$ was switched off (i.e., $x_{0}=0$ ) at $t=400,000$ and only the input $x_{1}$ was still active.

Figure 4 shows the weight development of $\rho_{1}$ over time for two different learning rates $\mu$. With a low learning rate, the weight $\rho_{1}$ approximately stabilizes when the input $x_{0}$ is switched off (see Figure 4a), whereas with a higher learning rate, the weight continues to grow. Weight stabilization can be very desirable during learning, but so is a high learning rate. These conflicting demands therefore lead to a trade-off, which needs to be taken care of in practical applications (like the robot application).

2.2.2 More Than One Filter in the Predictive Pathway. The setup with only one resonator $(N=1)$ in the predictive pathway has the disadvantage that there is only one specific temporal interval $T_{\text {opt }}$ where learning (weight change) is at the maximal rate. The use of an array of resonators with different frequencies in the predictive pathway removes this disadvantage (see the inset in Figure 5). The system should now be able to learn more than only one time interval properly. We have set up such a system with an array of 10 resonators in the predictive pathway. We triggered this array with the same $\delta$-pulse $\left(x_{1}=\delta(t)\right)$. The reflex pathway was triggered by a delayed 
$\delta$-pulse $\left(x_{0}=\delta(t-T) ; T=10\right)$. The initial condition for learning was set to $\rho_{0}=1 ; \rho_{k}=0 ; k \geq 1$ as before.

Signal shape. Figure 5 shows the resonator responses $u_{k}$ scaled with their momentarily existing weights $\rho_{k}$ (top) at time $t=390,000$ during learning. The scaled response of $u_{0}$ (a, dashed line) is still the biggest at this time. The diagram also shows the output signal $v$ and its derivative during the learning process (also $t=390,000$, bottom). Additionally, the output signal generated when silencing the input $x_{0}$ is shown (c, dotted line, bottom, $t=400,000)$.

The output $v$ is a superposition of all resonator outputs. It can be seen that it has a first and a second maximum (marked with 1 and 2 in Figure 5). The second maximum is due to the resonator response from the reflex pathway $u_{0}$ and vanishes when the input $x_{0}$ is switched off (see the dotted curve in c).

The first maximum is generated by superposition of the responses $\rho_{k} u_{k}$, $k>0$ (i.e., all except $u_{0}$ ). In general, we have observed that this superposition process will always try to generate the first maximum as close as possible to $x_{0}$. This can be understood by the ongoing amplification of an initially existing asymmetry in the system in the following way. At the first learning step, the derivative of $v$ is zero before $x_{0}$ and then follows the shape of the $v^{\prime}$ curve, as shown in the diagram. Thus, there is one resonator response whose shape matches the $v^{\prime}$ curve best (best positive correlation). Obviously, it is that particular resonator that has its maximum at (or closest to) the maximum of the $v^{\prime}$ curve (second cusp; the first is still zero). For this resonator, we get the highest correlation result (see equation 2.9) and, thus, the strongest weight growth at the beginning of learning. The other weights grow less strong, and their growth rate is approximately (inversely) related to the distance of their resonator maximum from $x_{0}$. As a consequence, we get a distribution of weight values that follows the shape outlined by the $y$-position of the resonator maxima, as shown in the top panel by the dots on the curves. Superposition of these weighted responses thus leads to a maximum of $v$ at $x_{0}$. This line of argumentation continues to hold for the following learning steps, because the theoretical results suggest that the contribution of the correlation of the first part of the $v^{\prime}$ curve (first cusp) with the $u_{k}, k>0$, which would correspond to homosynaptic learning, is zero in all cases (see equations 2.21-2.23), thereby not affecting the weight change. Thus, weight change continues to follow the distribution of the maxima in Figure 5a. The resonator with the lowest frequency $\left(f_{l}\right)$ determines the longest delay $T_{\max }=\frac{1}{f_{l}}$, which can be learned. Equivalently, the shortest delay is $T_{\min }=\frac{1}{f_{h}}$, where $f_{h}$ is the resonator with the highest frequency. Within the range $\left[T_{\min }, T_{\max }\right]$, any $T$ causes an output with a maximum that always coincides with the location of $x_{0}$, provided there are enough resonators to allow for a sufficiently accurate superposition process. 


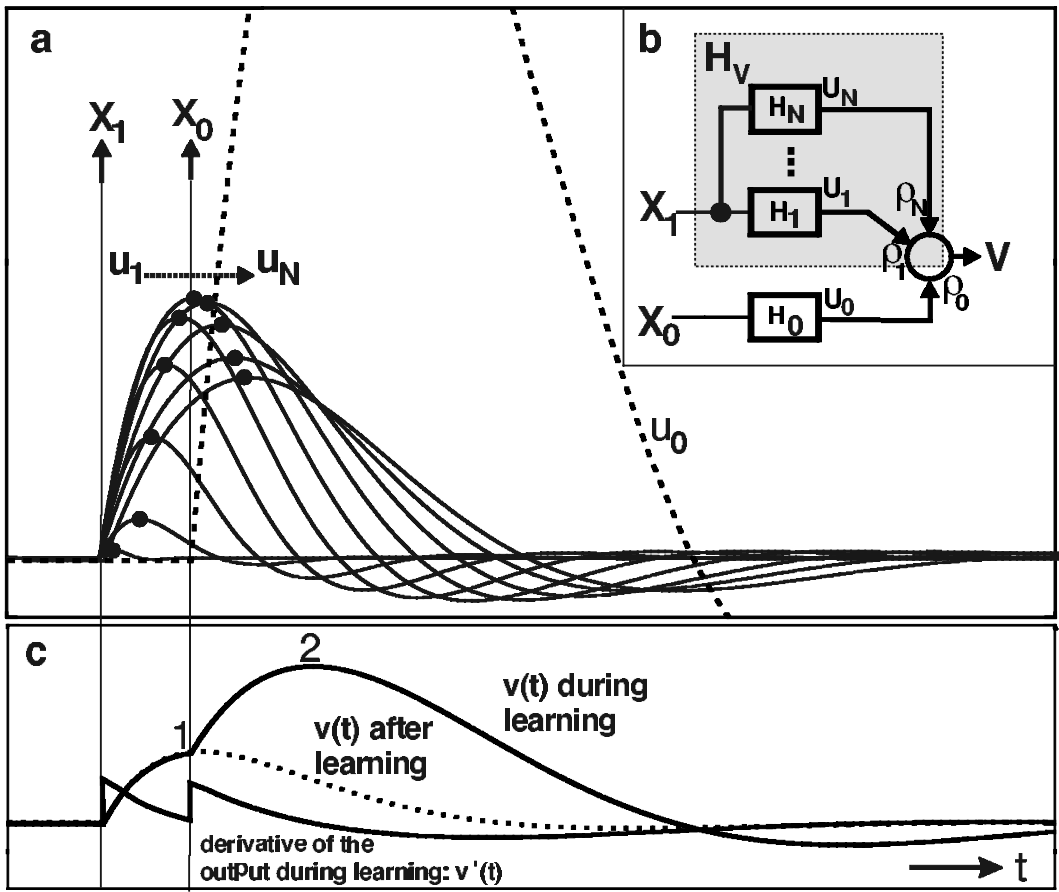

Figure 5: Multiple filters ( $N=10)$ in the predictive pathway: (a) Filter responses, (b) the neuronal circuit, and (c) its output during learning and after learning. The neuronal circuit $(b)$ consists of a filter bank where the filter frequencies are set to $f_{k}=\frac{5 f_{0}}{k} ; k \geq 1$ and $f_{0}=0.01$. The learning rate was set to $\mu=0.0005$ and $Q=1$. The filter bank gets two different inputs: $x_{0}(t)=\delta(t)$ (reflex pathway) and $x_{1}(t)=\delta(t-T)$ (predictive pathway), $T=10$. The delta pulses are repeated every 2000 time steps. After 400,000 time steps, $x_{0}$ is set to zero. The contribution of the signals $u_{k} \rho_{k}$ to the output $v$ triggered by $x_{1}(t)$ is called $H_{V}$ and is marked by the shaded box in $b$. The weighted resonator responses $\rho_{k} u_{k}$ after learning are shown in $a$. The output signals during learning (time step 390,000) and after learning (after time step 400,000) are shown in $c$.

Learning curve. As in the case of only two resonators, the dependence of the weight change on the temporal distance $T$ can be explored. Now, however, we have to monitor $N$ changeable weights. For this experiment, we have chosen the same standard setup using paired $\delta$-pulses with a temporal delay of $T$, but now we use 15 resonators $(N=15)$ in the predictive pathway. Their frequencies are chosen such that 10 resonators have a frequency that is higher and 5 resonators one that is lower than $f_{0}$ (see Figure 6). Every second weight change curve is shown in Figure 6 for $t=0$, where we varied 


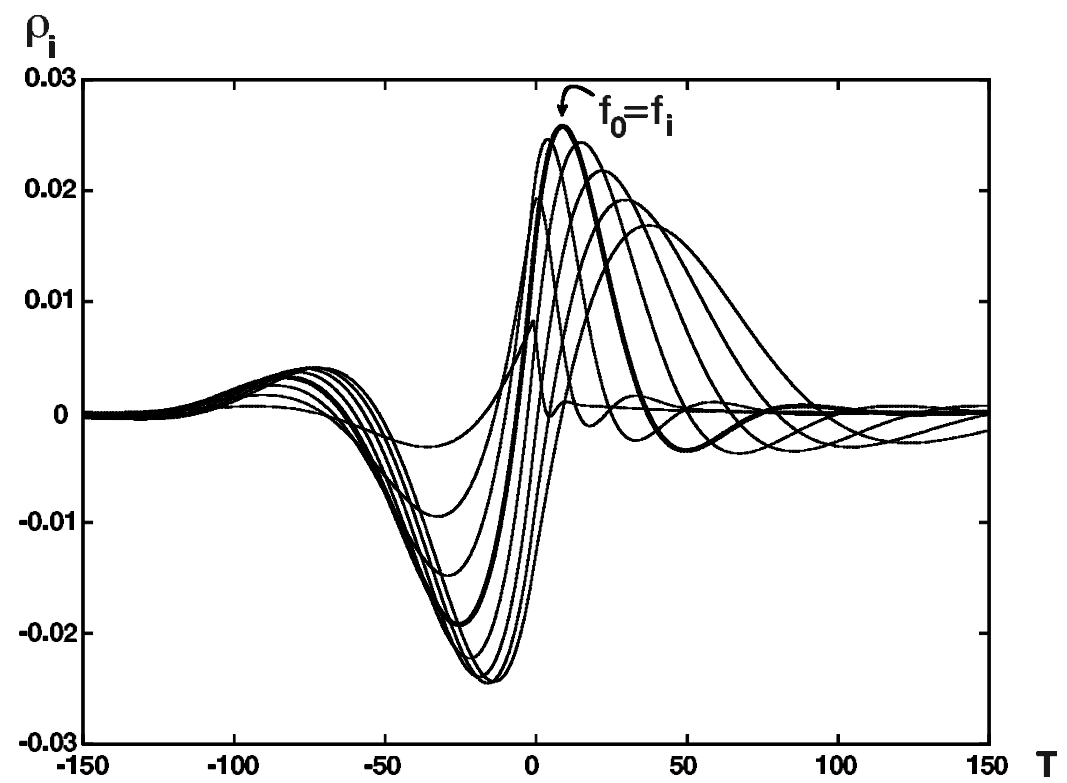

Figure 6: Weight changes $\rho_{j}$ dependent on the temporal distance $T$ with a filter bank of resonators $(N=15)$ set up as in Figure $5 \mathrm{~b}$. The filter frequencies are set to $f_{k}=\frac{5 f_{0}}{k} ; k \geq 1$ with $f_{0}=0.01$ and $Q=1$. The learning rate was set to $\mu=0.0001$ and $Q=1$. The case $f_{0}=f_{k}$ is marked with a thick line and reproduces the curve in Figure 2b. The filter bank gets two different inputs: $x_{1}(t)=\delta(t)$ (predictive pathway) and $x_{0}(t)=\delta(t-T)$ (reflex pathway). The delta pulses are repeated every 2000 time steps. After 400,000 time steps, the weight $\rho_{j}$ was measured and plotted against the temporal difference $T$. Only every second curve is plotted.

$T$ from -150 to 150 . Every curve in this diagram represents one weight $\rho_{k}$ of a specific resonator $h_{k}$ in dependence of $T$. The curve plotted with the thick line belongs to the resonator $h_{k}$, which has the same frequency as the resonator $h_{0}$, hence, $f_{k}=f_{0}$. The other weight change curves belong to resonators in the predictive pathway, which have different frequencies compared to $f_{0}$. It can be seen that every weight change curve has a specific $T$ where weight change is maximal, or (in support of the argument used to explain the first maximum in Figure 5) the other way around: for specific values of $T$ and large $N$, there always exists one particular resonator that shows maximum weight change.

Another interesting result is that the weight change curve with $f_{k}=f_{0}$ is identical to the weight change curve with only one resonator (see Figure 6). The fact that both weight change curves are the same is due to the linearity of our model. 

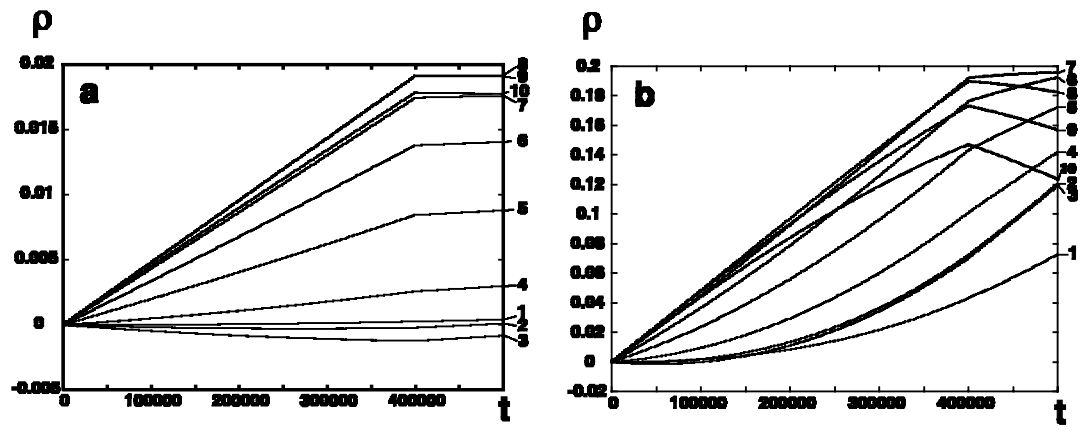

Figure 7: Weight change of multiple resonators $N=10$ in dependence of the learning rate. The neuronal circuit (see Figure $5 b$ ) consists of a filter bank where the filter frequencies are set to $f_{k}=\frac{0.1}{k} ; k \geq 1$, where the index $k$ is also used as a label for the different curves in this figure $(Q=1$ in both cases). The filter bank gets two different inputs: $x_{1}(t)=\delta(t)$ (predictive pathway) and $x_{0}(t)=\delta(t-T)$ (reflex pathway) with $T=10$. The delta pulses are repeated every 2000 time steps. After 400,000 time steps, $x_{0}$ was set to zero. The learning rate was set to (a) $\mu=0.0001$ and (b) $\mu=0.001$.

In summary, in an array of different resonators, every resonator is responsible only for a specific and limited range of temporal intervals so that such an array is able to cover a wide range of different temporal intervals. The weight change curves for the different weights give precise information as to which resonator yields the maximum contribution to the output signal.

Weight stabilization for $x_{0}=0$. Next, we ask whether the weights also stabilize in a multiresonator setup if the reflex pathway $x_{0}$ becomes zero (compare to Figure 7). We use the same setup as before $(N=10$ and paired $\delta$-pulses with $T=10$ ). The test was performed in the same way as above by setting $x_{0}$ to zero at time $t=400,000$. Figure 7 shows that the weights stabilize in the limit of $\mu \rightarrow 0$. Thus, we find again that the crucial parameter for an approximate weight stabilization is the learning rate $\mu$, which is too high in Figure $7 b$.

Because of the complexity of the mathematics, we were not able to give robust analytical arguments for weight stabilization in the multiresonator case. We could argue only that the individual resonator responses (sine waves) should be orthogonal to the derivative of the output (cosine wave) as soon as $x_{0}=0$ (see the dashed curve in Figure 6), leading to zero value of the correlation integral. The experimental findings in Figure 7 support this notion. Thus, in the multiresonator case, we obtain the desired property of weight stabilization in the limit of $\mu \rightarrow 0$. Homosynaptic learning does not take place even with more than two resonators. 
Let us in the context of $N>1$ also briefly consider more than one predictive pathway with several sensors that operate independently. In this case, the isotropy of the algorithm leads to the situation that learning will continue between those sensor inputs even after the reference (reflex) input $x_{0}$ has become silent. Weight changes of the other (nonreflex) weights, however, will normally remain small because after learning, the absolute values of them are small, which leads only to minor cross influences, as will be shown in the robot example (see Figure 11). Thus, even in such a situation, weights will be (approximately) stable.

Another stabilizing factor arises if we place the learning algorithm in a closed behavioral loop (see also the next section). In the closed-loop paradigm analyzed in our companion article, we found that a perturbation of the weight $\rho_{1}$ (which disturbs the final condition $x_{0}=0$ ) leads to a counterforce that reestablishes the original weight. Taken together, these arguments show that weights might indeed undergo small drifts after removal of the reflex, but these drifts do not lead to a divergence. This is supported by the robot experiments, where we never observed weight divergence even after prolonged runs.

\section{Closed Loop: The Robot Experiment}

The task in this robot experiment is collision avoidance. The built-in reflex behavior is a retraction reaction after the robot has hit an obstacle (see Figure 8 , solid pathway). This represents a typical feedback mechanism with the desired state that the signal at the collision sensor should remain zero. In order to prevent the robot from leaving the desired state, it can use other sensor modalities that can predict a looming collision. In our case, this is achieved with range finders (see Figure 8, dashed pathway). The learning algorithm has the task of learning the existing temporal correlation between the range finder and the collision sensor signals. After learning, the robot can generate a motor reaction in response to the range finder signals and thereby avoid the retraction reflex. Functionally, the reflex will be eliminated, and the "predictive pathway" takes over after learning.

Up to this point, the algorithm had been treated in a pure open-loop condition, where learning was entirely unsupervised. The robot experiments create a situation where the behavioral reaction influences the sensor inputs, thereby creating a closed-loop situation (see Figure 8). Unsupervised learning thereby turns into something that we would call self-referenced learning in order to distinguish it from reinforcement learning, which requires an explicitly defined reference signal (punishment or reward), which is not present in closed-loop ISO learning. The theoretical treatment of this situation in our companion article will clarify that these two situations are fundamentally different.

The robot's circuit diagram is shown in Figure 9; a detailed description, which includes a list of the robot's control parameters, is given in 


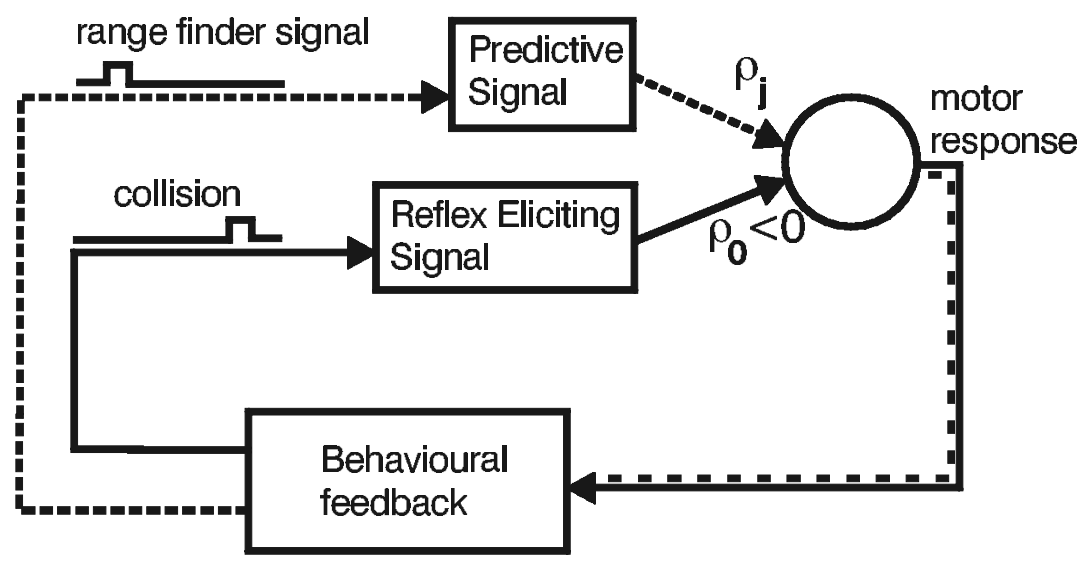

Figure 8: Simple sensor motor feedback with prediction that is made explicit with the example of collision avoidance. The solid lines depict a prewired reflex loop that exists before learning. This reflex loop performs a reflex reaction-in this case, a retraction reaction (motor response) when the collision sensor (reflex eliciting signal) has been triggered. The task is to learn that the earlier range finder signal (predictive signal, dashed pathway) can be used to generate an earlier motor reaction to prevent the collision (reflex).

appendix $C$. The robot has three collision sensors and two range finders. All signals are filtered by bandpass filters and converge onto two neurons, which generate two different motor outputs: one controls the robot's speed and the other the robot's steering angle. The speed of the robot is set to a fixed value and its steering to zero so that the undisturbed robot drives straight forward. The built-in retraction behavior is generated by the dotted pathways where the collision sensors trigger highly damped sine waves in the corresponding resonators. This signal is sign inverted and directly transmitted to the motors. Essentially, it consists of just a single half-wave, which leads to the retraction reaction. The weights are initially set to minus one and effectively do not change during learning, so that the retraction behavior always remains the same. The dotted collision sensor pathways with their strong weights that determine the motor output are, together with the arising behavioral feedback, equivalent to the reflex loop discussed in Figure 8.

The range finder signals (solid lines) react at a distance of about $15 \mathrm{~cm}$ from an obstacle and are therefore able to predict a collision. However, the temporal delay between the range finder signal and the collision signal is variable and depends on the actual motion trajectory of the robot. In order to cope with a rather wide range of temporal delays, we used the same approach as in section 2.2.2 and implemented two resonator filter banks, which get their signals from the two range finders. Filter banks consist of 10 


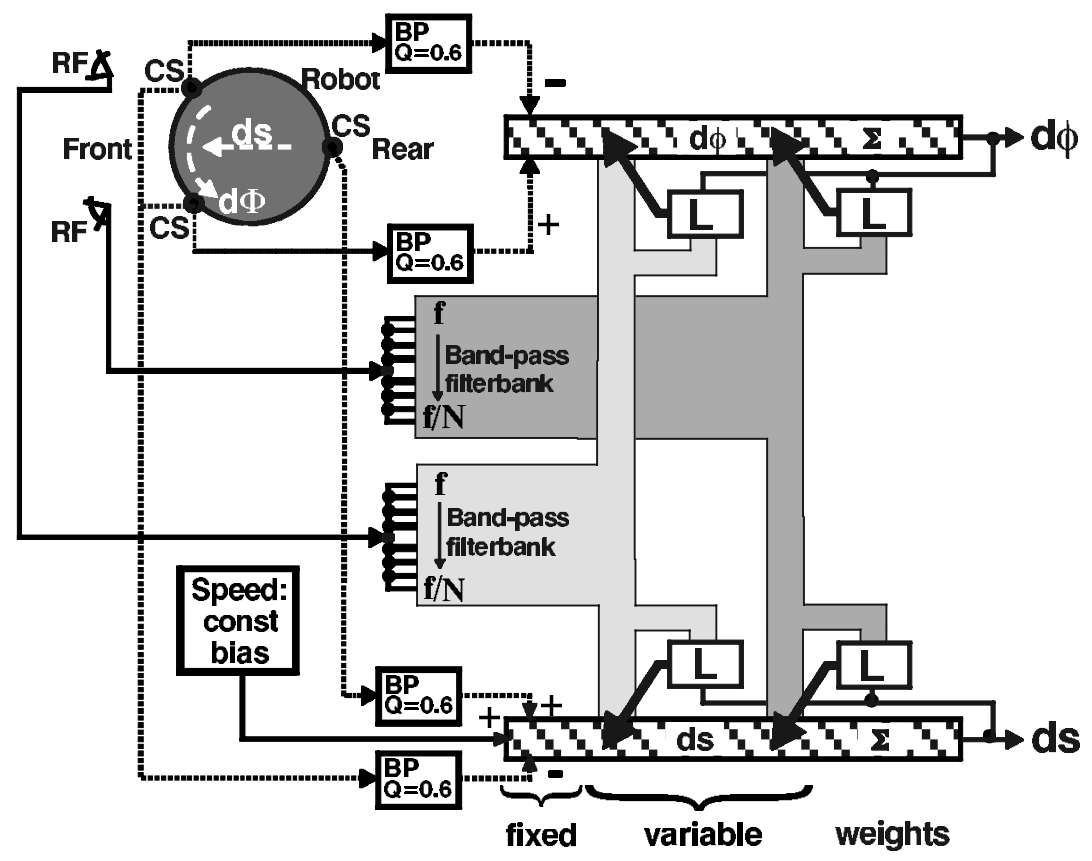

Figure 9: Robot circuit: The robot consists of three collision sensors (CS), two range finders (RF), and two output neurons for speed $(d s)$ and steering angle $(d \phi)$. These output neurons represent simple summation circuits (indicated by $\sum$ ). The robot has a reflex behavior established by the signals from the collision sensors (dotted lines), which are fed into four bandpass filters $H_{0}$ with $f_{0}=1 \mathrm{~Hz}$ and $Q_{0}=0.6$. The output of the bandpass filters is summed at the neurons for speed $(d s)$ and steering angle $(d \phi)$. The corresponding weights are adjusted in such a way that the robot performs an appropriate retraction reaction if either of the collision sensors is triggered. The task of learning is to use the signals from the range finders (RF) to predict the trigger of the collision sensor (CS). The two signals from the left and the right range finder are fed into two filter banks, with $N=10$ resonators with frequencies of $f_{k}=\frac{1 \mathrm{~Hz}}{k} ; k \geq 1$ and $Q=1$ throughout. The 20 signals from the two filter bank converge on both the speed neuron and the neuron responsible for the steering angle. Learning rate was $\mu=0.00002$. $L$ depicts the implementation of the learning rule (see equation 2.2).

resonators covering approximally a temporal interval between $50 \mathrm{~ms}$ and $500 \mathrm{~ms}$. These resonator signals converge onto both the speed neuron and the steering neuron. Their weights are initially set to zero.

Depending on the initial conditions, the robot found different solutions to avoid obstacles. One solution, for example, is that after learning, the robot simply stops in front of an obstacle or slightly oscillates back and forth. This 
type of behavior may look trivial but is entirely compatible with the learning goal of avoiding obstacles. More commonly, we observed a different type of solution where the robot continuously drives around and uses mainly its steering to generate avoidance movements. Other solutions do not seem to be possible and have not been observed. Furthermore, we observed that the robot always found one of these solutions after sufficiently long learning.

Figure 10 shows episodes of the robot behavior and its signals for one selected example trajectory. The signals shown in Figures 10c and 10d correspond to a situation where the robot still collides with the walls. Corresponding collision points are marked in Figure 10a by $c$ and $d$. As expected, learning leads to a change of the temporal relation between the range finder signal and the collision signal. This can be seen by the different lengths of $T$ depicted in Figures 10c and 10d and is due to the learned motor output, which is increasingly dominated by the range finder signal. This supports the filter bank approach, which we have used in the robot experiment. Finally, Figure 10e depicts a situation where the robot has learned to avoid the obstacles $(C S=0)$.

Note that the low-pass component of the bandpass filters smoothes the rater noisy range finder signals, which substantially adds to the robustness of the algorithm. Furthermore, pure noise signals are not correlated to other sensor signals and do not contribute to learning.

The change of the weights in the robot example shall now be compared with the results from the simulations. We find that the weights approximately stabilize in our robot experiments (see Figure 11). Their actual values depend on the solution found. The situation in the robot experiment, however, is more complicated than in the simulations shown earlier, because the $d s$ - and $d \phi$-neurons get signals from more than two sensors at the same time. Thus, often triplets of temporal correlations exist; for example, during a slanted wall approach, we first obtain a signal from the right, then one from the left range finder, and finally one from the right collision sensor. After successful learning, the collision sensor remains silent, but we are left with sequences of range finder events. Thus, learning continues, though at a smaller rate, even after the last collision.

As a central observation, this shows that our system continues to operate without a designated reference signal (because $x_{0}$ is zero now). Learning continues between the remaining inputs. This can be seen in Figure 11 when looking at the development of the weight from the left range finder to $d \phi$, which continues to change after the last collision has occurred (at $t=85 \mathrm{~s}$ ). Ultimately, the earlier of the two range finder signals would dominate, but this will lead to a stable situation only for very simple (e.g., circular) trajectories, where an unchanging relation between both range finder signals is forced on the robot.

An equivalent reward-retrieval situation has also been simulated. These results shall not be presented here in order to limit the length of this article but can be viewed on-line at http://www.cn.stir.ac.uk/predictor/animat/. 

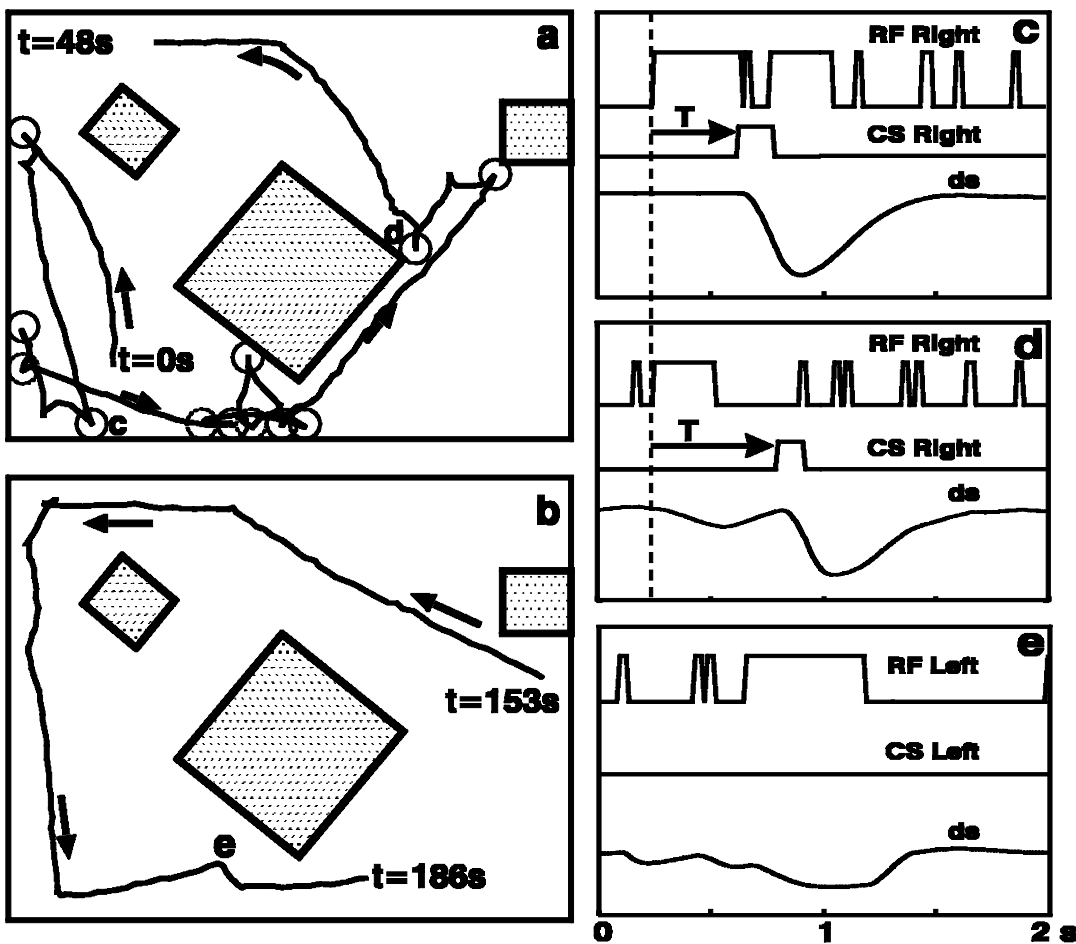

Figure 10: (a) Manually reconstructed robot movement trace in an arena $(240 \mathrm{~cm} \times 200 \mathrm{~cm})$ with three obstacles (shaded) at the onset of learning. Motors were not entirely balanced, leading to a curved start of the trajectory. Many collisions (solid circles show forward collision and dashed circles backward collision) occur, and trapping at obstacles happens. After a collision, a fast reflexlike retraction and turning reaction is elicited. (b) Robot movement trace after successful learning of the temporal correlation between signals at RF and CS. No more collisions occur; the trajectory is smooth. A complete movie of this trial can be viewed at http://www.cn.stir.ac.uk/predictor/real-movie 1. (c-e) Signals at RF (top), CS (middle), and motor control signal $d s$ (bottom) for different learning stages. (c) Signals occurring at the early collision marked $c$ in part $a$. A stereotyped motor reaction is elicited in response to the CS signal. (d) Signals occurring at the late collision $d$. Motor reactions occur in response to RF but are not sufficient to avoid the collision. When it occurs, a strong motor reaction is again elicited. (e) Signals occurring at the curve marked $e$ in part $b$. Smooth motor reactions occur in response to RF; CS remains silent because no collision occurs. 

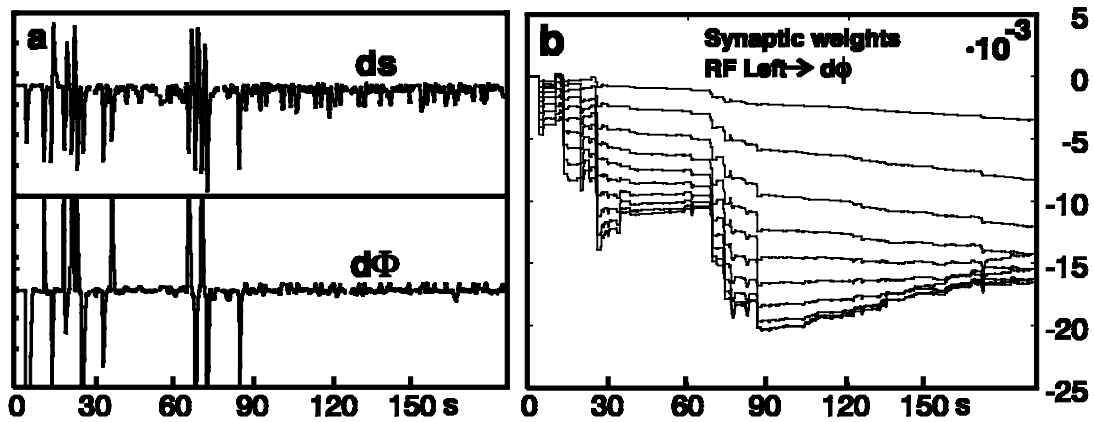

Figure 11: (a) Complete motor signal traces for $d s$ and $d \phi$ and (b) development of the synaptic weights for the same trial as in Figure 10.

\section{Discussion}

In this study, we have developed an isotropic algorithm for sequence order learning (ISO learning) in which learning relies only on the temporal order of its inputs. This has the advantage that all input signals are treated equally and that learning takes place between all of them. Thus, it represents a form of unsupervised sequence order learning.

4.1 Basic Properties. ISO learning is driven only by the temporal relation between pre- and postsynaptic signals. As a consequence, our learning rule is related to learning based on spike-timing-dependent plasticity (STDP; Gerstner, Kempster, van Hemmen, \& Wagner, 1996; Gerstner, Kreiter, Markram, \& Herz, 1997; Markram et al., 1997; Zhang et al., 1998; Bi \& Poo, 1998; Roberts, 1999; Xie \& Seung, 2000; Kistler \& van Hemmen, 2000; Song, Miller, \& Abbott, 2000; Song \& Abbott, 2001; Fu et al., 2002), but our algorithm uses time-continuous functions and not spike trains as input signals. The measured curves for STDP are based on the relation between individual (pre- and postsynaptic) spikes. Curves with different characteristic shapes have been observed (Abbott \& Nelson, 2000), such as that similar to our Figure 2, but also inverted versions of it have been measured. Thus, the question arises how these curves relate to the average firing rate of the neurons (Gerstner et al., 1997). This question was specifically addressed in the studies of Roberts (1999) and Xie and Seung (2000), who found that the temporal derivative of the postsynaptic impulse rate directly relates to the temporal Hebbian STDP curve, or with sign inversion to the anti-Hebbian curve. This shows that a computational link exists between rate-based ISO learning and the spike-based STDP results because we found that the Hebbian STDP curve will be obtained analytically by integrating the ISO learning rule over time (see equations 2.10 through 2.14). 
In the second part of this study, we introduced a closed-loop situation by means of behavioral feedback. We implemented a primary reflex loop, which is distinguished from all other inputs only by the fact that it initially carries the largest synaptic weight. In general, such closed-loop reflex loop situations have the disadvantage that any reaction will occur only after an incoming sensor event. This inherent disadvantage of feedback loops leads to a general objective for improving animal behavior, which is to find a mechanism that prevents the reflex (Palm, 2000; Wolpert \& Ghahramani, 2000). Sequence order learning can achieve this by creating earlier, anticipatory actions. In addition, we have shown that weights stabilize as soon as the reflex has been successfully avoided. Due to the isotropy of the inputs, any other input line can take on the role of the reference signal during learning, and the initial reflex can even be unlearned or reduced in strength, a situation observed in many physiological reflexes.

4.2 Practical Aspects. A convenient aspect of ISO learning that leads to a very limited computational effort is the use of infinite impulse response filters in our approach. With such filters, it is possible to generate a smooth and long-lasting response when using only two resonators. Such a response can bridge a very long temporal difference $T$ and is therefore able to generate a basic predictive reaction. Additional filters contribute to increasingly precise timing. Thus, the basic temporal correlation between $X_{1}$ and $X_{0}$ can be established by one filter $(N=1)$ and then improved by adding more and more filters. In other approaches (Sutton \& Barto, 1981, 1988; Klopf, 1988), delay lines are often used for the predictive input $X_{1}$. The discrete structure of these algorithms requires many more delay elements as compared to the analog operating ISO learning because they need a delay element for every unit time-step. Thus, the computational effort is much higher if a broad temporal range has to be covered.

4.3 Evaluative versus Nonevaluative Models. A fundamental difference exists between reference-based (reward-based) algorithms (e.g., TD learning) and so-called drive reinforcement algorithms, such as differential Hebbian learning and ISO learning.

Probably the most influential method for reference-based temporal sequence learning is the TD learning algorithm (Sutton, 1988). TD learning has the goal of generating an output $v$, which predicts a reference (reward $r$ ) by the help of its (sensorial) input signals $x$. This goal is achieved by minimizing a prediction error $\delta$ between reference and output. Thus, learning relies on the predefined reference, which acts like a teacher signal in supervised learning.

The direct comparison between the two algorithms shows that the reference (reward) pathway and the error calculation of TD learning are replaced by the reflex pathway in our algorithm. Mathematically, the reflex pathway is not functionally distinct from the other pathways in ISO learning; 
however, it drives the output with an initially strong weight. As described in section 2.2.1, the strongest input dominates the learning behavior of the other inputs and weights. Klopf (1988) called this drive reinforcement learning. In appendix $B$, we compare the different drive reinforcement models in the literature. Here, we just note that our algorithm belongs to the class of pure unsupervised learning algorithms opposed to TD learning, which is supervised using the reference (reward) as teaching signal. The initially strong weight in the reflex pathway of ISO learning can be interpreted as a boundary condition, preventing the output from becoming arbitrary. Introducing boundary conditions is typical practice of unsupervised, especially Hebbian, learning (Miller, 1996).

The structural differences of our learning algorithm and TD learning suggest different neuronal substrates. The TD learning circuitconsists of two components: the predictive circuit and the error signal circuit. Usually, these two circuits are identified with different neuronal subsystems: the error circuit with the dopamine system and the predictive circuit with cortical or other dopamine-modulated brain areas. Strong supporting evidence is found in Schultz et al. (1997), and it is also known that reward-based learning plays a substantial role in animal behavior, such as during instrumental (operand) conditioning paradigms and action planning (Dayan \& Abbott, 2001).

Our algorithm, on the other hand, suggests only one neuronal circuit because all pathways are equivalent, as supported by Hauber, Bohn, and Grietler (2001). It is conceivable that such a system coexists with the rewardbased learning systems, because in an autonomous agent, any reward-based system needs to be bootstrapped by first correlative experiences, such as those used by our system to drive learning.

In the one-circuit scenario, our learning rule, based on temporal relation between pre- and postsynaptic signals, would have to be represented by internal neuronal variables like NMDA dynamics or $\mathrm{Ca}^{2+}$ concentration. The direct relation of our learning rule with the shape of the STDP curves (see Figure $2 b$ ) indicates that it should be relatively straightforward to redesign our model into a biophysically more realistic one, which directly relies on such internal neuronal variables and uses spike trains as inputs. This has recently been attempted by Rao and Sejnowski (2001) using the TD learning algorithm but the relation between TD learning and STDP is less direct, and, accordingly, the transition between those two models is bit more intricate (Dayan, 2002).

When talking to specialists in the field of temporal sequence learning, we were asked to explain to what degree our learning rule is different from the one used in TD learning. This aspect is quite technical, and we refer readers to appendix $B$ for an in-depth discussion.

4.4 Closed-Loop Condition. Hebbian learning rules like the one used here belong to the class of unsupervised learning rules. Unsupervised learn- 
ing seems to be the obvious choice for creating the first and earliest stages of autonomous behavior, because it does not require external (teacher-like) knowledge. Instead, it relies purely on self-organization based on the correlation structure of the inputs. Such unguided self-organization processes, however, can also lead to a situation where nonsensical correlations are learned, leading in the end to an undesired network behavior. The standard solution to avoid this problem is the introduction of boundary conditions, which keep the self-organization process within sensible margins. In practice, this is done either heuristically by the network designer or, as a better choice, boundary conditions are introduced such that they intrinsically (and in a natural way) represent the structure of the problem to which the selforganization process is applied.

In the case of our unsupervised temporal sequence learning algorithm, the same is achieved by embedding the learning circuit in an environment that leads to a closed-loop situation. The causal relation that naturally exists between many different pairs of sensor events (e.g., pain follows heat, taste follows smell) as described in section 1 creates an implicit boundary condition for our algorithm by using the latest incoming event (the one that drives the reflex) as the temporal reference for learning. The environment has two properties in our model: it provides feedback, and it contains disturbances, but very clearly it does not provide any reward or any other teaching signal. Klopf (1988) called this feedback loop nonevaluative since there is nothing in the environment that evaluates the organism's performance. Instead, here ISO learning becomes self-referenced (von Foerster, 1960; Maturana and Varela, 1980): the actions of the learner influence its own learning without any evaluation process.

Robotics is the discipline that can clarify the concepts of autonomous behavior and interaction with a complex environment quite naturally (Brooks, 1997). In the field of temporal sequence learning, Vershure has been working for over 10 years in using robot applications (Verschure \& Pfeifer, 1992; Verschure \& Voegtlin, 1998). In his words, every organism undergoes three steps of development: prewired reflex (fixed connections), adaptive control (classical Hebbian learning of sequences of sensor inputs), and reflective, contextual control (goal-oriented learning). In Vershure's terminology, adaptive control has no goals but builds up temporal associations with "proximal" and "distal" sensors. At the stage of the reflective control, a goal is introduced in the form of a reward or punishment when, for example, an object has successfully been found.

Our study shows that this distinction may be too rigid. The behavioral pattern observed in our robot seems to be punishment guided, which would place it at the advanced level of reflective control. The unsupervised, nonevaluative, but self-referenced structure of the robot's interaction with the environment, however, places it at the simpler level of adaptive control. This shows that autonomous agents can develop rather complex behavioral patterns by means of simple nested feedback loop systems, without hav- 
ing to evaluate their own behavior. Of course, we would not argue against the importance of higher learning schemes, and it is also quite sensible to distinguish between increasingly higher levels starting with nonevaluative schemes, which are surpassed by evaluative (reward and punishment) schemes, which are followed up by contextual learning and finally by the different stages of cognitive learning. But it seems advisable to treat these different stages in a less separatist way, allowing for a broader transition range between them.

In our companion article, we derive a theoretical treatment of the closedloop ISO learning situation. We will show analytically that the predictive pathway learns to approximate the inverse controller of the reflex pathway, thereby creating a forward model of the control situation.

\section{Appendix A: Plancherel's Theorem}

This theorem is not widely known, so we state it here as

$$
\begin{aligned}
\int_{0}^{\infty} f_{1}(t) f_{2}(t) d t & =\frac{1}{2 \pi} \int_{-\infty}^{+\infty} F_{1}(i \omega) F_{2}(-i \omega) d \omega \\
& =\frac{1}{2 \pi} \int_{-\infty}^{+\infty} F_{1}(-i \omega) F_{2}(i \omega) d \omega
\end{aligned}
$$

where $F$ is the Laplace transform of $f$ (Stewart, 1960). If we set $f_{1}=f_{2}=f$, it becomes the more commonly used theorem of Parseval.

\section{Appendix B: Comparison Between TD, ISO Learning, and Other Differential Hebbian Learning Algorithms}

One has to distinguish drive reinforcement models such as those by Sutton and Barto (1981), Klopf (1986, 1988), and Kosco (1986) (to which ISO learning also belongs) from reference-based reinforcement models such as TD learning (Sutton, 1988; Dayan \& Sejnowski, 1994).

We first discuss the differences between the different drive reinforcement models, which are shown in Figures 12a through 12c. The central difference between ISO learning and the other techniques is that in ISO learning, all inputs are filtered before they are summed at the output neuron. In the other algorithms, the inputs are summed in an unfiltered way. A low-pass filter is applied only to the conditioned stimulus when it enters the learning pathway (i.e., before the correlator $\times$ ). This leads to a fundamentally different behavior of ISO learning because as a result, ISO learning produces an orthogonal behavior between input and output (after filtering, the inputs resemble sine waves; thus, the derivative of the output resembles a sum of cosines; see Figure 2). This orthogonal behavior, which crucially relies on the filtering of all pathways, leads to the inherent and desired 
a) Sutton \& Barto, 1981

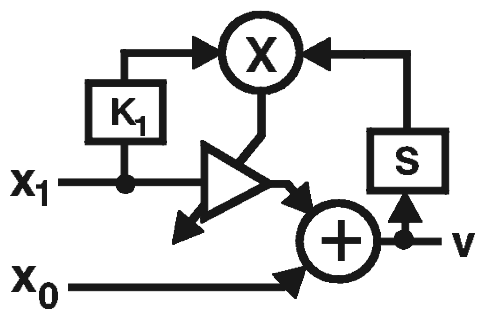

c) ISO-learning

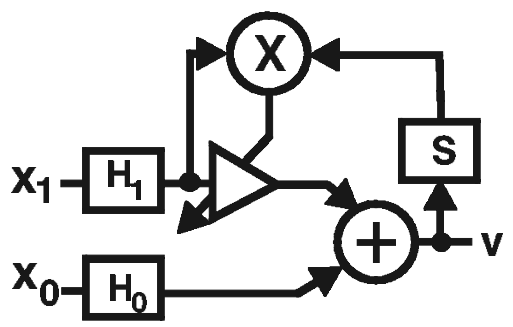

b) Klopf, Kosco, 1986

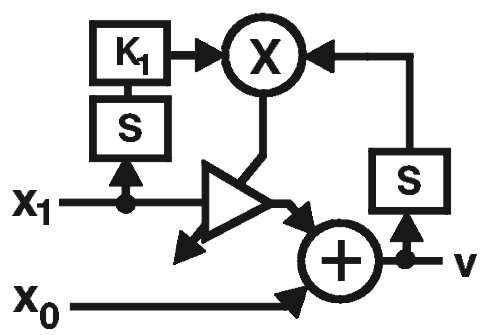

d) Sutton and Barto,1988 TD-learning

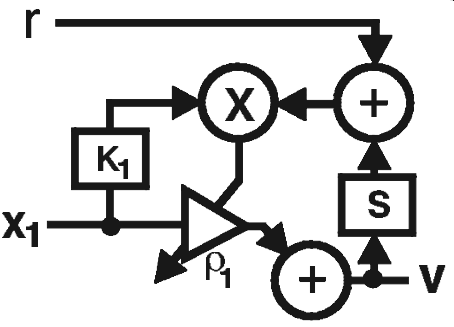

Figure 12: Comparison of $(\mathrm{a}-\mathrm{c})$ three drive reinforcement algorithms and (d) TD learning in Laplace notation. Transfer functions are denoted as $H, K$, and the derivative operator as $s . X_{0}$ represents the unconditioned and $X_{1}$ the conditioned input. The amplifier symbol denotes the changing synaptic weight. Note that $c$ is drawn with a fixed weight at $X_{0}$ to make it more easily comparable to the other diagrams. All models use a derivative of the postsynaptic signal in order to control the weight change. Both Sutton and Barto models $(a, d)$ use low-pass filters $K$ only in the conditioned pathway. Klopf's model $b$ is identical to model $a$ with the exception of an additional temporal derivative at $x_{1}$. Only in ISO learning are all inputs filtered, which together with the output-derivative generates orthogonal behavior, leading to weight stabilization. For further explanation, see the text.

weight stabilization property of ISO learning, which does not arise in the other drive-reinforcement algorithms without additional measures taken. Furthermore, we note that Klopf has introduced an additional derivative at the conditioned input, because he focuses on signal changes.

TD learning belongs to yet another category of sequence learning algorithms. The difference arises from the fact that TD is evaluative (reference based; see Figure 12d), whereas the drive reinforcement models operate in a nonevaluative way. We have already discussed this elementary difference 
at great length. Here, we focus on the aspect that TD learning also uses some kind of derivative, which suggests a strong structural similarity between TD and ISO learning methods. In spite of this apparent similarity, however, our approach is more strongly related to Kalman filtering than to TD learning. This is due to the combination of linear filtering and applying a derivative. The predictive property of our algorithm thereby arises from the fact that every low-pass filtered function is smooth, which leads to the situation that its derivative linearly predicts its future development. This property of low-pass filtered signals is well known in signal theory and is mainly used in the Kalman filter theory (Bozic, 1994).

TD learning instead calculates a temporal difference error $\delta$ (similar to the famous $\delta$-rule by Widrow \& Hoff, 1960) by means of subtracting subsequent output values from each other and relating this error value to the reward: $\delta(t)=r(t)+v(t+1)-v(t)$. The second group of terms seems to be related to the derivative used in our approach. This mathematical similarity, however, carries a distinctively different interpretation, which can be understood as follows: The goal of TD learning is that the output $v(t)$ at any point in time should predict the total remaining reward,

$$
v(t)=\sum_{s \geq t}^{T} r(s),
$$

at the end of learning. Take the example of a rat exploring a maze where at each intersection, a decision about a turn has to be made, creating a temporal sequence of events. Each turn leads to a different reward (e.g., food) to be picked up along the way. This clarifies the concept of total remaining reward until the end of the maze is reached at $T$. Furthermore, it is known that the total remaining reward can be iteratively approximated using the next following prediction value $v(t+1)$ to yield something like the total remaining expected reward:

$$
\sum_{s \geq t}^{T} r(s) \approx r(t)+v(t+1):=e(t, t+1) .
$$

During learning, this total remaining expected reward $e$ is compared with its actual prediction $v$ in order to define the prediction error $\delta$. Thus, $\delta(t)=$ $e(t, t+1)-v(t)$, leading to the apparent similarity of the resulting temporal difference terms $v(t+1)-v(t)$ in TD learning with the derivative we used. From this interpretation, however, it is quite clear that the term $v(t+1)$ arises only in conjunction with $r(t)$. This kind of conjunction cannot be found in our algorithm because it is reward free. Furthermore, the structure of TD learning is acausal, looking forward in time using $v(t+1)$ to calculate $\delta(t)$. This and the reward-based structure of TD learning make it rather difficult to associate it with STDP, as attempted by Rao and Sejnowski (2001) 
(see Dayan, 2002 for discussion). Thus, the formal similarities between both rules do not seem to warrant treating them as equal. These interpretations continue to hold for the time-continuous version of TD learning designed by Doya (2000).

\section{Appendix C: The Robot}

- Hardware. A modified commercial robot ("rug warrior," $16 \mathrm{~cm}$ diameter) was used. Two active wheels are driven by DC motors, and steering is achieved through different DC levels. Average speed was adjusted to $0.45 \mathrm{~m} / \mathrm{s}$ using a control parameter $c=0.6$. In order to detect mechanical contact, the robot has three microswitches, $C S_{l}, C S_{r}, C S_{b}$, in a triangular configuration (see Figure 9) Visual signals are generated by two multiplexed, infrared-emitting, active range finders $R F_{l}, R F_{r}$ with an angle of 70 degrees between them. Infrared reflection is detected by an infrared sensor centered between the emitters, which operates in synchrony with them. The detection range was adjusted to 0.5 to $15.0 \mathrm{~cm}$. Interfacing between robot and computer is done tethered via a conventional I/O card.

- Sensor characteristics. Sensor signals are bandpass filtered, as in many biological systems. This is achieved by feeding the raw signal into a bandpass with transfer function $h(t)$. The output functions of the bandpass filters are denoted as $u_{k}$ and normalized to one. The bandpass characteristics of all collision sensors $h_{r}(t)$ are identical with $Q=0.6$ and $f=1 \mathrm{~Hz}$. The signal from each vision sensor is fed in parallel into a filter bank of 10 bandpass filters. Its frequencies are set to $f_{k}=\frac{10}{i} \mathrm{~Hz}, k=1,2, \ldots, 10 ; Q$ is set to 1.0 throughout. The filter bank approach assures that large and varying temporal intervals between vision sensor and collision sensor signals can be covered.

- Neuronal circuitry. The robot has two neurons: one that controls the speed $d s$, the other the steering angle $d \phi$. Normal operation is straightforward motion ( $d s=$ const, $d \phi=0)$. Both neurons receive inputs from all sensors in a direct feedforward connectivity.

- Unconditioned retraction reaction. The unconditioned retraction reaction uses only the collision sensor signals. These signals drive the output neurons in such a way that an avoidance movement with a motion vector pointing away from the site of stimulation is elicited.

- Learning. All bandpass filter outputs $u_{k}$ from the collision and the vision sensors converge onto both neurons, where they are summed according to their synaptic weights $\rho_{k}$. The change of the weights is achieved by learning rule equation 2.2. The constant $\mu$ is set to 0.00002 .

- Neuronal Output (resulting from unconditioned retraction reaction and learning). The output of the neurons is defined as $d s=c-\rho_{0}^{d s}\left[h_{r}(t) *\right.$ $\left.\left(C S_{l}+C S_{r}-C S_{b}\right)\right]+l_{d s}$ and $d \phi=\rho_{0}^{d \phi}\left[h_{r}(t) *\left(C S_{l}-C S_{r}\right)\right]+l_{d \phi}$. The asterisk 
denotes a convolution operation. The variables $l_{d s}$ and $l_{d \phi}$ represent the total sum of all learned contributions that converge onto the $d s-$ and $d \phi$ neuron, respectively. Learning follows equation 2.2. The synaptic weights in unconditioned reaction are initially set to $\rho_{0}^{d s}=0.15$ and $\rho_{0}^{d \phi}=-0.5$.

\section{Acknowledgments}

We are grateful to Christian von Ferber, Leslie Smith, Richard Reeve, and the members of the CCCN seminar for their helpful comments during various stages of this work. This study was supported by grants from SHEFC RDG INCITE and by the European funding ECOVISION.

\section{References}

Abbott, L., \& Blum, K. (1996). Functional significance of long-term potentiation for sequence learning and prediction. Cereb. Cortex, 6, 406-416.

Abbott, L., \& Nelson, S. B. (2000). Synaptic plasticity: Taming the beast. Nature Neuroscience (Suppl.) 3, 1178-1179.

Ashby, W. R. (1956). An introduction to cybernetics. London: Methuen.

Bi, G.-q., \& Poo, M.-m. (1998). Synaptic modifications in cultured hippocampal neurons: Dependence on spike timing, synaptic strengh, and postsynaptic cell type. J. Neurosci., 18(24), 10464-10472.

Bozic, S. M. (1994). Digital and Kalman filtering: An introduction to discrete-time filtering and optimum linear estimation. London: E. Arnold.

Brooks, R. A. (1989). How to build complete creatures rather than isolated cognitive simulators. In K. VanLehn (Ed.), Architecturesfor intelligence (pp. 225-239). Hillsdale, NJ: Erlbaum.

Brooks, R. A. (1997). Intelligence without representation. In H. John (Ed.), Mind design II (pp. 395-420). Cambridge, MA: MIT Press.

Dayan, P. (2002). Matters temporal. Trends in Cognitive Sciences, 6(3), 105-106.

Dayan, P., \& Abbott, L. F. (2001). Theoretical neuroscience. Cambridge, MA: MIT Press.

Dayan, P., Kakade, S., \& Montague, P. R. (2000). Learning and selective attention. Nature Neuroscience (Suppl.) 3, 1218-1223.

Dayan, P., \& Sejnowski, T. (1994). TD $(\lambda)$ converges with probability 1. Mach. Learn., 14(3), 295-301.

Doya, K. (2000). Reinforcement learning in continuous time and space. Neural Networks, 12(1), 219-245.

Fu, Y.-X., Djupsund, K., Gao, H., Hayden, B., Shen, K., \& Dan, Y. (2002). Temporal specificity in the cortical plasticity of visual space representation. Science, 296, 1999-2003.

Gerstner, W., Kempter, R., van Hemmen, L., \& Wagner, H. (1996). A neural learning rule for submilisecond temporal coding. Nature, 383, 76-78.

Gerstner, W., Kreiter, A. K., Markram, H., \& Herz, A. V. (1997). Neural codes: Firing rates and beyond. Proc. Natl. Acad. Sci. USA, 94, 12740-12741. 
Grossberg, S. (1995). A spectral network model of pitch perception. J. Acoust. Soc. Am., 98(2), 862-879.

Grossberg, S., \& Merrill, J. (1996). The hippocampus and cerebellum in adaptively timed learning, recognition and movement. J. Cogn. Neurosci., 8, 257-277.

Grossberg, S., \& Schmajuk, N. (1989). Neural dynamics of adaptive timing and temporal discrimination during associative learning. Neural Networks, 2, 79-102.

Guo-Quing, B., \& Poo, M.-M. (1998). Synaptic modifications in cultured hippocampus neurons. J. Neurosci., 18(24), 10464-10472.

Haruno, M., Wolpert, D. M., \& Kawato, M. (2001). Mosaic model for sensorimotor learning and control. Neural Comp., 13, 2201-2220.

Hauber, W., Bohn, I., \& Grietler, C. (2001). NMDA, but not dopamin $\mathrm{D}_{2}$ receptors in the rat nucleus accumbens are involved in guidance of the instrumental behaviour by stimuli predicting reward magnitude. J. Neurosci., 20(16), 6282-6288.

Hebb, D. O. (1949). The organization of behavior: A neuropsychological study. New York: Wiley-Interscience.

Kandel, E., Abrams, T., Bernier, L., Carew, T., Hawkins, R., \& Schwartz, J. (1983). Classical conditioning and sensitization share aspects of the same molecular cascade in Aplysia. Cold Spring Harb. Symp. Quant. Biol., 48(2), 821-830.

Kistler, W. M., \& van Hemmen, J. L. (2000). Modeling synaptic plasticity in conjunction with the timing of pre- and postsynaptic action potentials. Neural Comp., 12, 385-405.

Klopf, A. H. (1986). A drive-reinforcement model of single neuron function. In J. S. Denker (Ed.), Neural networks for computing: AIP conference proceedings (Vol. 151). New York: American Institute of Physics.

Klopf, A. H. (1988). A neuronal model of classical conditioning. Psychobiol., 16(2), 85-123.

Kosco, B. (1986). Differential Hebbian learning. In J. S. Denker (Ed.), Neural networks for computing: AIP conference proceedings(Vol. 151). New York: American Institute of Physics.

Levy, W. B., \& Minai, A. A. (1993). Sequence learning in a single trial. In Proceedings of the 1993 INNS World Congress on Neural Networks II (pp. 505-508). Hillside, NJ: Erlbaum.

Markram, H., Lübke, J., Frotscher, M., \& Sakman, B. (1997). Regulation of synaptic efficacy by coincidence of postsynaptic APs and EPSPs. Science, 275, 213-215.

Maturana, H., \& Varela, F. J. (1980). Autopoiesis and cognition: The realization of the living. Dordrecht: Reidel.

McGillem, C. D., \& Cooper, G. R. (1984). Continuous and discrete signal and system analysis. New York: CBS Publishing.

Miller, K. D. (1996). Receptive fields and maps in the visual cortex: Models of ocular dominance and orientation columns. In E. Donnay, J. van Hemmen, \& K. Schulten (Eds.), Models of neural networks III (pp. 55-78). New York: Springer-Verlag. 
Montague, P. R., Dayan, P., \& Sejnowski, T. J. (1993). Foraging in an uncertain environment using predictive hebbian learning. In J. D. Cowan, G. Tesauro, \& J. Alspector (Eds.), Advances in neural information processing systems, 6 (pp. 598-605). San Mateo, CA: Morgan Kaufmann.

Oja, E. (1982). A simplified neuron model as a principal component analyzer. J. Math. Biol., 15(3), 267-273.

Palm, W. J. (2000). Modeling, analysis and control of dynamic systems. New York: Wiley.

Rao, R. P., \& Sejnowski, T. J. (2001). Spike-timing-dependent Hebbian plasticity as temporal difference learning. Neural Comp., 13, 2221-2237.

Roberts, P. D. (1999). Temporally asymmetric learning rules: I. Differential Hebbian learning. J. Comput. Neurosci., 7(3), 235-246.

Saudargiene, A., Porr, B., \& Wörgötter, F. (2003). Biophysical evaluation of a linear model for temporal sequence learning: ISO-Learning revisited. Manuscript in preparation.

Schultz, W., Dayan, P., \& Montague, P. R. (1997). A neural substrate of prediction and reward. Science, 275, 1593-1599.

Schultz, W., \& Suri, R. E. (2001). Temporal difference model reproduces anticipatory neural activity. Neural Comp., 13(4), 841-862.

Shepherd, G. M. (Ed.). (1990). The synaptic organisation of the brain. New York: Oxford University Press.

Song, S., \& Abbott, L. (2001). Column and map development and cortical remapping through spike-timing dependent plasticity. Neuron, 32, 339-350.

Song, S., Miller, K. D., \& Abbott, L. F. (2000). Competitive Hebbian learning through spike-timing-dependent synaptic plasticity. Nature Neuroscience, 3, 919-926.

Stewart, J. L. (1960). Fundamentals of signal theory. New York: McGraw-Hill.

Sutton, R. (1988). Learning to predict by method of temporal differences. Machine Learning, 3(1), 9-44.

Sutton, R., \& Barto, A. (1981). Towards a modern theory of adaptive networks: Expectation and prediction. Psychol. Review, 88, 135-170.

Sutton, R., \& Barto, A. (1988). Simulation of anticipatory responses in classical conditioning by a neuron-like adaptive element. Behav. Brain. Res., 4(3), 221-235.

Traub, R. D. (1999). Fast oscillations in cortical circuits. Cambridge, MA: MIT Press.

Verschure, P. F., \& Pfeifer, R. (1992). Categorization, representations, and the dynamics of system-environment interaction: A case study in autonomous systems. In H. Roitblat, J. Meyer, \& S. Wilson (Eds.), Proceedings of the Second International Conference on Simulation of Adaptive Behaviour (pp. 210-217). Cambridge, MA: MIT Press.

Verschure, P., \& Voegtlin, T. (1998). A bottom-up approach towards the aquisition, retention, and expression of sequential representations: Distributed adaptive control III. Neural Networks, 11, 1531-1549.

von Foerster, H. (1960). On self-organizing systems and their environments. In M. Yovits \& S. Cameron (Eds.), Self-organizing systems (pp. 31-50). New York: Pergamon Press. 
Widrow, G., \& Hoff, M. (1960). Adaptive switching circuits. IRE WESCON Convention Record, 4, 96-104.

Wolpert, D. M., \& Ghahramani, Z. (2000). Computational principles of movement neuroscience. Nature Neuroscience (Suppl.), 3, 1212-1217.

Xie, X., \& Seung, S. (2000). Spike-based learning rules and stabilization of persistent neural activity. In S. A. Solla, T. K. Leen, \& K.-R. Müller (Eds.), Advances in neural information processing systems, 12 (pp. 199-208). Cambridge, MA: MIT Press.

Zhang, L. I., Tao, H. W., Holt, C. E., Harris, W. A., \& Poo, M.-M. (1998). A critical window for cooperation and competition among developing retinotectal synapses. Nature, 395, 37-44.

Received April 12, 2002; accepted October 28, 2002. 